\title{
SÍNTESE DE NOVAS TIOSSEMICARBAZONAS ISOXAZOLÍNICAS AZA-BICÍCLICAS DE SEIS MEMBROS, COM POTENCIAL ATIVIDADE ANTICHAGÁSICA
}

J. A. B. MESQUITA ${ }^{1}$, E. K. SOUZA ${ }^{1}$, F. N. COUTINHO ${ }^{1}$, M. S. De ARAÚUJO NETA ${ }^{1}$, L. N. De BARROS ${ }^{1}$, M. C. Q. De ARAÚJO ${ }^{1}$, A. C. Da SIlva Jr. ${ }^{1}$, D. R. Da SILVA ${ }^{1}$, A. R. De FARIA ${ }^{1 *}$

${ }^{1}$ Universidade Federal de Pernambuco, Centro de Ciências da Saúde, Departamento de Ciências Farmacêuticas, Laboratório de Síntese Orgânica Aplicada a Fármacos - LASOF.

E-mail para contato: mesquita22@live.com, ${ }^{*}$ rodolfo.ufpe@gmail.com

RESUMO - Utilizando o método de hibridização molecular, inéditas tiossemicarbazonas-isoxazolínicas aza-bicíclicas de seis membros foram sintetizadas. As enamidas endocíclicas de 6 membros, utilizadas como material de partida, foram obtidas a partir da acilação da 1-piperideína (trímero da 1-piperideína). Posterior cicloadição 1,3-dipolar das enamidas encocíclicas com o $\mathrm{N}$-óxido de nitrila-CEFNO, isoxazolinas aza-bicícliocas de 6 membros foram obtidas como cicloadutos, tendo a função éster em posição C3. Subsequente redução do éster com $\mathrm{NaBH}_{4}$ ao respectivo álcool e posterior oxidação do álcool em C3, via Swern, os aldeídos isoxazolínicos foram obtidos. A última etapa consistiu na condensação dos aldeídos isoxazolínicos com diferentes tiossemicarbazidas adquiridas comercialmente. As moléculas hibridas finais, assim como intermediários inéditos foram caracterizados através de espectroscopia de RMN ${ }^{1} \mathrm{H}, \mathrm{RMN}{ }^{13} \mathrm{C}$ e IVFT e tiveram também algumas de suas propriedades físico-químicas determinadas.

Palavras Chaves: tiossemicarbazonas, isoxazolinas aza-bicíclicas, antichagásicos

ABSTRACT-. Unpublished six-membered thiosemicarbazones-aza-bicyclic isoxazolines were synthesized using the molecular hybridization method. The 6-membered endocyclic enamides, used as starting material, were obtained from the acylation of 1-piperideine (1-piperideine trimer). 1,3-dipolar cycloaddition of the endocyclic enamides with the nitrile oxide - CEFNO, 6-membered aza-bicyclic isoxazolines were obtained as cycloadducts, showing ester function in the C3 position. Subsequent reduction of the ester with $\mathrm{NaBH}_{4}$ to the respective alcohol and following oxidation of the alcohol at $\mathrm{C} 3$ via Swern, the isoxazoline aldehydes were obtained. The last step consisted of condensation of the isoxazoline aldehydes with different, commercially acquired thiosemicarbazides. The final hybrid molecules, tiosemicarbazone / isoxazoline, as well as novel intermediates were characterized by ${ }^{1} H N M R,{ }^{13} C N M R$ and IVFT spectroscopy and also had some of their particular physicochemical properties

Keywords: thiosemicarbazones, aza-bicyclic isoxazolines , antichagasic 


\section{INTRODUÇÃ̃O}

A hibridização molecular, uma importante ferramenta na química medicinal, consiste na junção de fragmentos bioativos (farmacóforos) em uma única estrutura química, sendo uma estratégia clássica e eficiente para o desenho de novos protótipos (BARREIRO, et al., 2003). A conjugação de características estruturais de duas ou mais classes distintas de compostos em uma nova molécula, é a essência da seção química deste trabalho.

Existe a necessidade da síntese de novas moléculas com potencial atividade antichagásica, já que só existem dois fármacos (Benznidazol e Nifurtimox) com aplicabilidade clínica comprovada e ambos apresentam atividade significativa apenas na fase aguda da doença, com até $80 \%$ de cura parasitológica, na fase crônica a eficácia é relativa. Estima-se que a doença afete cerca de 8 a 10 milhões de pessoas em todo o mundo, principalmente na América Latina, tornando-se um sério problema global (COURA; J. R.; DIAS, J. C. P, 2009).

Logo, este trabalho tem por objetivo a síntese de novas moléculas híbridas, contendo o núcleo 2-isoxazolina aza-bicíclica de seis membros e tiossemicarbazonas, com potencial atividade antichagásica, que estão sendo avaliadas.

Figura 1 - Molécula híbrida contendo os núcleo 2-isoxazolina azabicíclica de seis membros e a tiossemicarbazona.

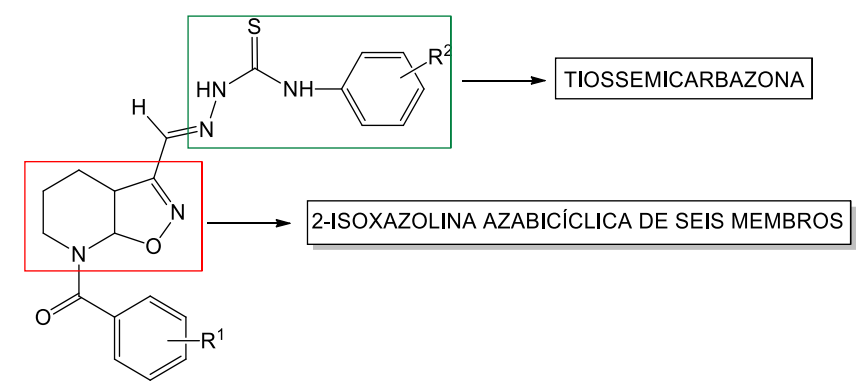

\section{MATERIAIS E MÉTODOS}

O Esquema 1 apresenta a rota de síntese das novas tiossemicarbazonas isoxazolínicas de seis membros (ㄱ) . A piperidina (1) , obtida comercialmente, é oxidada à 1-piperideína, através da eliminação da $N$-cloropiperidina em meio básico. Por apresentar instabilidade, a 1-piperideína, trimeriza no meio reacional, formando o trímero da 1-

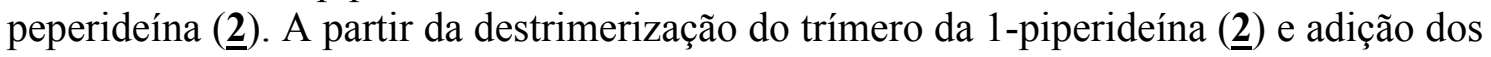
agentes acilantes (cloretos de benzoila) obteve-se as enamidas endocíclicas de seis membros ()ㅡ, que são os dipolarófilos na etapa seguinte. A reação de cicloadição 1,3dipolar das enamidas endocíclicas ( $\underline{\mathbf{3}}$ ) com o óxido de carboetoxiformonitrila (CEFNO), propiciou a obtenção dos cicloadutos isoxazolínicos de seis membros (4), que sofreram subsequente redução do grupamento éster em $\mathrm{C} 3$, via borohidreto de sódio $\left(\mathrm{NaBH}_{4}\right)$, obtendo-se os álcoois isoxazolínicos de seis membros (므). Estes por meio da reação de Swern sofreram oxidação branda, produzindo os respectivos aldeídos isoxazolínicos de seis membros (ㅁ). (REIS; DE FARIA, 2011)

Por fim, foram realizadas as condensações dos aldeídos isoxazolínicos (ㅁ) com diferentes tiossemicarbazidas, obtidas comercialmente, produzindo as inéditas tiossemicarbazonas isoxazolínicas de seis membros (ㄱ). Nesta última etapa, foi utilizado o etanol (P.A.) como solvente e o ácido clorídrico concentrado como catalizador da reação. 
Esquema 1: Rota sintética para obtenção das novas tiossemicarbazonas isoxazolínicas aza-bicíclicos de seis membros ․ 7. a) i) $\mathrm{AcOH}$ glacial,ii) $\mathrm{N}$-clorosuccinimida/KOH b) cloretos de benzoila, Et $\left.t_{3} \mathrm{~N}, T H F, \boldsymbol{c}\right) \mathrm{Et}_{3} \mathrm{~N}$, Clorooxiimidoacetato de etila, $\mathrm{THF}$ d) $\left.\mathrm{NaBH}_{4}, \mathrm{MeOH}, \boldsymbol{e}\right) \mathrm{DMSO}, \mathrm{TEA}$, $\left.\mathrm{C}_{2} \mathrm{O}_{2} \mathrm{Cl}_{2}, \mathrm{CH}_{2} \mathrm{Cl}_{2}, \boldsymbol{f}\right)$ Tiossemicarbazidas

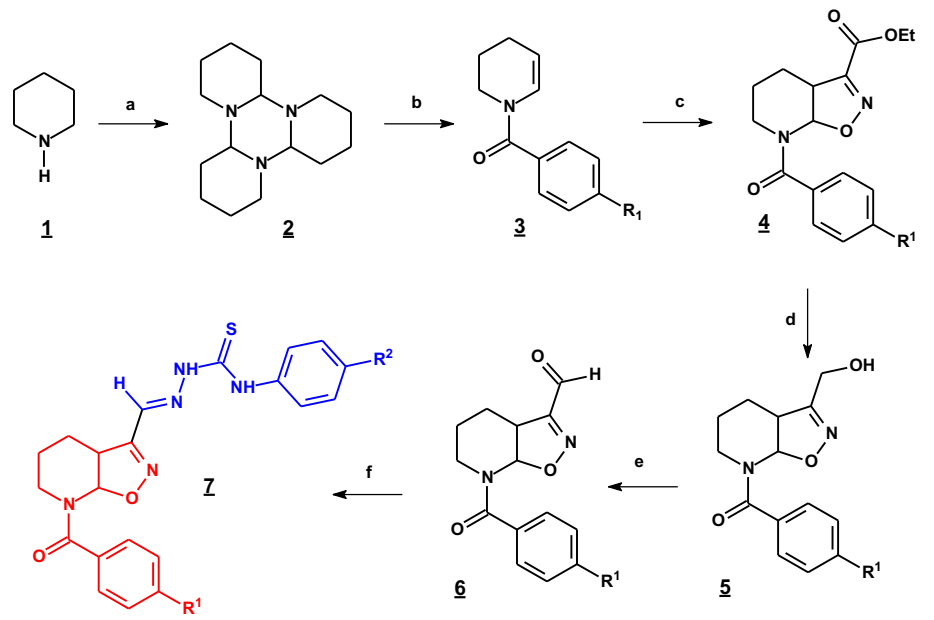

\section{RESULTADOS E DISCUSSÃO}

A tabela 1 apresenta algumas propriedades físico-químicas das inéditas tiossemicarbazonas isoxazolínicas aza-bicíclicas de seis membros $\underline{\mathbf{7}}$, como rendimentos e fator de retenção em cromatografia em camada fina. As tiossemicarbazonas isoxazolínicas foram devidamente caracterizadas por meio das espectroscopias de $\mathrm{RMN}$ de ${ }^{1} \mathrm{H}$, de $\mathrm{RMN}{ }^{13} \mathrm{C}$ e IVFT . Os espectros de ${ }^{1} \mathrm{H}$ RMN de $\underline{\mathbf{7 a}}$ e $\underline{7 \mathbf{b}}$ são apresentados representativamente nas figuras 2 e 3 , mostrando, entre outros, os sinais "diagnósticos" da formação dos heterobicíclos isoxazolínicos, que são os hidrogênios dos metinos da junção dos anéis, ligado aos átomos de oxigênio e nitrogênio, com deslocamentos químicos próximos de $6,00 \mathrm{ppm}$ e 4,00 ppm respectivamente, assim como os hidrogênios HN-N em torno de 12 ppm e HN-ph em torno de $10 \mathrm{ppm}$, confirmando as estruturas propostas no planejamento das novas moléculas. Os espectros de ${ }^{13} \mathrm{C}$ RMN e no IVFT também corroboram as análises feitas em ${ }^{1} \mathrm{H}$ RMN.

Tabela 1 - Identificação e dados físico-químicas das semicarbazonas isoxazolínicas.

\begin{tabular}{|c|c|c|c|c|c|c|c|}
\hline Código & $\mathrm{R}_{1}$ & $\mathrm{R}_{2}$ & Fórmula & Aspecto & $\begin{array}{c}\text { Rf AcOEt:Hex } \\
(3: 2)\end{array}$ & P.F. & Rendimento \% \\
\hline$\underline{\underline{7 a}(\mathrm{AJ} 04)}$ & $\mathrm{OCH}_{3}$ & $\mathrm{H}$ & $\mathrm{C}_{22} \mathrm{H}_{23} \mathrm{~N}_{5} \mathrm{O}_{3} \mathrm{~S}$ & pó amarelado & 0,51 & $207^{\circ} \mathrm{C}-208^{\circ} \mathrm{C}$ & 54 \\
\hline$\underline{7 \mathbf{b}}(\mathrm{AJ} 03)$ & $\mathrm{CH}_{3}$ & $\mathrm{H}$ & $\mathrm{C}_{22} \mathrm{H}_{23} \mathrm{~N}_{5} \mathrm{O}_{2} \mathrm{~S}$ & pó amarelado & 0,59 & $213^{\circ} \mathrm{C}-216^{\circ} \mathrm{C}$ & 62 \\
\hline
\end{tabular}

Figura 2 - Espectro de $\mathrm{RMN}{ }^{1} \mathrm{H}\left(300 \mathrm{~Hz}, \mathrm{DMSO}, \mathrm{DMSO}, 35^{\circ} \mathrm{C}\right) \mathrm{de} \underline{\mathbf{7}}^{\mathbf{a}}$.

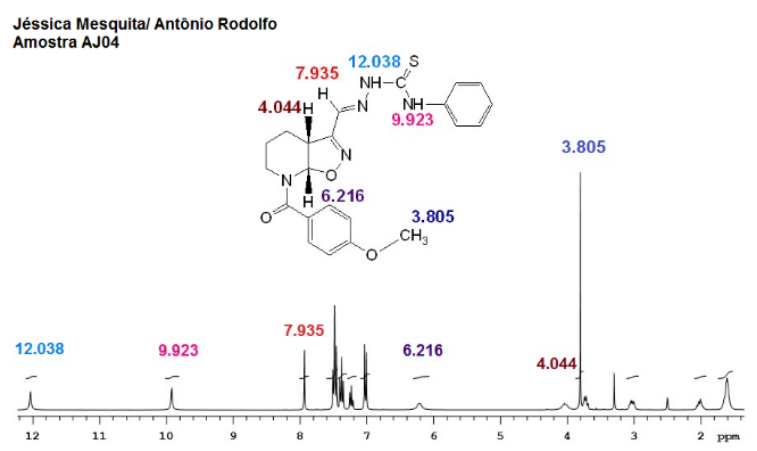


Figura 3 - Espectro de $\mathrm{RMN}{ }^{1} \mathrm{H}\left(300 \mathrm{~Hz}, 35^{\circ} \mathrm{C}\right)$ de $\underline{7 \mathbf{b}}$.

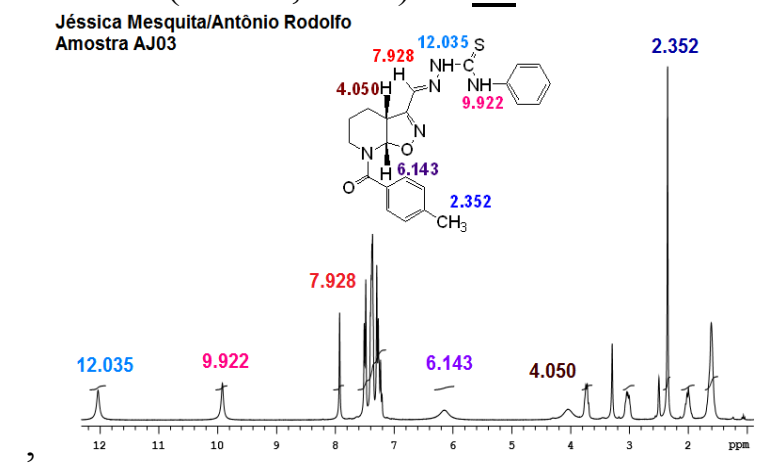

\section{CONCLUSÃO}

As novas tiossemicarbazonas isoxazolínicas aza-bicíclicas de seis membros $\underline{7}$ foram sintetizadas e elucidadas quimicamente, assim como tiveram suas características físicoquimicas determinadas. O estudo antichagásico in vitro está em andamento.

\section{REFERÊNCIAS}

1- BARREIRO, E. J.; FRAGA, C. A. M.; MIRANDA, A. L. P.; RODRIGUES, C. R.; Química Nova, 25, 129, 2003.

2- COURA; J. R.; DIAS, J. C. P. Epidemiology, control and surveillance of Chagas disease - 100 years after its Discovery. Mem Inst Oswaldo Cruz, v. 104, p. 31-40, 2009 3- REIS, S. L. G. B.; DE ALMEIDA, V. M., DE ALMEIDA G. C.; BOAVIAGEM, K. M.; MENDES, C. C. B.; GÓES, A. J. S.; MAGALHÃES, L. R.; DA SILVA, T. G.; DE FARIA, A. R.; Química Nova, 34, 76, 2011.

\section{AGRADECIMENTOS}

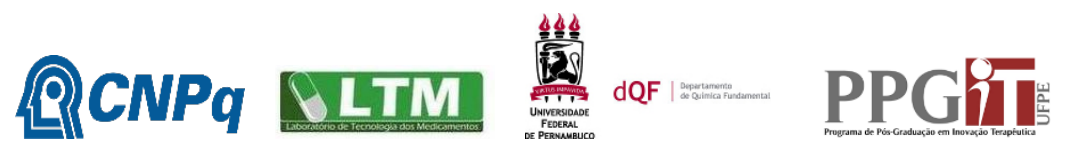

